\title{
MODELING OCULAR LENS DISEASE IN XENOPUS
}

Justine Viet , David Reboutier , Serge Hardy , Salil A. Lachke , Luc Paillard ${ }^{\mathrm{a}}{ }^{\mathrm{a}}$, , Carole GautierCourteille

a Univ Rennes, CNRS, IGDR-UMR 6290, F-35000 Rennes, France

b Department of Biological Sciences, University of Delaware, Newark, DE, USA

* Corresponding authors, LP (luc.paillard@univ-rennes1.fr) and CGC (carole.gautier@univrennes1.fr)

\section{RUNNING TITLE}

Xenopus ocular lens

\section{KEYWORDS}

Cataract; CRISPR; Pathology; GJA8; PAX6; DNASE2B

\section{JLLET POINTS}

- Ocular lens development is similar in Xenopus and in mammals

- Xenopus embryos disrupted for human cataract genes develop cataract-like lens defects

- Xenopus embryos with cataract have abnormal lenses and reduced visual acuity

- Xenopus embryos are a novel relevant model of lens development and disease

\section{GRANTS}

Accepted Articles are accepted, unedited articles for future issues, temporarily published online in advance of the final edited version. 
LP is supported by a grant from Bouygues and the Association Retina France, and a CNRS-PICS. SAL is supported by NIH Grants R01EY021505, R01EY029770. 


\section{ABSTRACT}

\section{Background}

Ocular lens clouding is termed as cataract, which depending on the onset, is classified as congenital or age-related. Developing new cataract treatments requires new models. Thus far, Xenopus embryos have not been evaluated as a system for studying cataract.

\section{Results}

We characterized the developmental process of lens formation in Xenopus laevis tailbuds and tadpoles, and we disrupted the orthologues of three mammalian cataract-linked genes in F0 by CRISPR/Cas9. We assessed the consequences of gene inactivation by combining external examination with histochemical analyses and functional vision assays. Inactivating the key metazoan eye development transcription factor gene pax6 produces a strong eye phenotype including an absence of eye tissue. Inactivating the genes for gap-junction protein and a nuclease, gja8 and dnase2b, produces lens defects that share several features of human cataracts, including impaired vision acuity, nuclei retention in lens fiber cells, and actin fibers disorganisation. We tested the potential improvement of the visual acuity of gja8 crispant tadpoles upon treatment with the molecular chaperone 4-phenylbutyrate.

\section{Conclusion}

Xenopus is a valuable model organism to understand the molecular pathology of congenital eye defects, including cataracts, and to screen molecules with a potential to prevent or reverse cataracts. 


\section{INTRODUCTION}

The ocular lens is an avascular structure in the eye whose function is to refract and focus light on the retina ${ }^{1}$. A major property of lenses is their transparency, and lens clouding, or cataract, is the leading cause of blindness worldwide. While most cataracts are age-related, there exist congenital cataracts, which can be genetic ${ }^{2}$. Their incidence is 1 to 6 children per 10,000 births $^{3-5}$. Cataracts are cured by replacing opaque lenses by artificial intraocular lenses. Despite generally excellent outcomes of cataract surgery, post-operative complications can occur, such as capsule rupture and loss of vitreous humour, infections, endophthalmitis or glaucoma. At a longer term, posterior capsular opacification (secondary cataract) results from the proliferation of epithelial cells that remain in the capsular sac after lens removal ${ }^{6,7}$. The annual number of patients with complications is far from negligible considering the high frequency of cataract surgeries. Cataract therefore remains a major public health issue, and a better understanding of the pathophysiological mechanisms that cause it as well as the quest for innovative treatments are highly important ${ }^{8}$.

While there are several models for cataract research, they vary in their strengths and weaknesses. 2D cell culture models only recapitulate a tiny part of the molecular events leading to cataracts. A 3D cell culture model (lens organoid) was recently developed as a promising in vitro model ${ }^{9}$. Animal models of cataract are the zebrafish "cloche" mutants, which tend to have lens defects suggestive of cataracts ${ }^{10}$, and mammals like rodents, rabbits or dogs ${ }^{11}$. For ethical and cost reasons, these models cannot be considered for large-scale studies like drug screening.

Xenopus embryos have several advantages as models of human pathologies. They develop externally, and raising hundreds of Xenopus embryos is highly cost-effective. The high number of ıased embryos ensures unequalled statistical power of the observations. Xenopus are tetrapods, hence evolutionary closer to humans than other small multicellular models like flies, worms, or even fishes. Xenopus is amenable to F0 reverse genetics, i.e. analyses of phenotypes of larvae developed from genetically modified embryos without any requirement for time-consuming crosses. However, only a few articles deal with cataract in Xenopus. Knocking-down sparc with antisense morpholinos in Xenopus results in a high mortality due to defective cell-cell adhesion after gastrulation, but surviving tailbuds injected with a reduced amount of morpholino display poorly characterised lens phenotypes evocative of cataract ${ }^{12}$. Knocking-down the orthologues of 
mammalian cataract genes like TMEM114, CHRLD1, SIPAL3 or CELF1 produces eye phenotypes generally loosely related to cataract ${ }^{13-16}$. Hence, whether ocular lens developmental defects, including cataract, can be modelled in Xenopus remains an open question. A prerequisite to modelling cataract is to have a good understanding of normal lens development. A stage series of Xenopus lens development was proposed decades ago ${ }^{17}$. More recent articles investigate Xenopus lens development, but are limited to early stages up to Nieuwkoop and Faber stage 38-40 ${ }^{18,19}$. In another article, these early stages are compared with a much older stage 50 embryo, but intermediate stages are not described ${ }^{20}$. Lens regeneration following lensectomy has also been extensively investigated in amphibians. In Xenopus, lens regeneration was divided into 5 stages characterized by precise cellular and molecular events, and much has been learnt from regeneration studies regarding lens development. However, lens development and lens regeneration are two different phenomena. During development, the lens placode originates from the preplacodal ectoderm whereas it results from cornea transdifferentiation during lens regeneration ${ }^{21-24}$. Furthermore, gene expression patterns differ between lens development and regeneration ${ }^{20,25}$. Hence, while Xenopus lens regeneration and development share many properties, the underlying cellular and molecular mechanisms are not necessarily the same. These observations call for completing the anatomical description of Xenopus lens development. We first describe lens morphogenesis during Xenopus laevis development. Next, to test if cataract can be detected in Xenopus larvae, we disrupt pax6, gja8 and dnase2b by CRISPR/Cas9-mediated genetic engineering. We selected these genes because they are associated with eye defects or cataract in mammals, and can potentially also be associated with lens defects in amphibians. We investigate the consequences of gene inactivation by combining morphological examination with histochemical analyses and functional vision assays. Disrupting these genes leads to eye phenotypes with variable severity that include lens defects that share several features of cataract in humans. We discuss the potential interest of genetically modified Xenopus larvae to screen molecules that may prevent or cure cataracts and to understand the molecular pathobiology of congenital cataracts.

\section{RESULTS}

\section{Lens development in Xenopus laevis}


We made a series of sections of Xenopus embryos to generate a developmental time series reference for normal lens development (Figure 1). During early stages of vertebrate development, the presumptive lens ectoderm thickens to form the lens placode, which invaginates ${ }^{1,26}$. In Xenopus, this results in an internalised poorly structured cell mass or lens rudiment ${ }^{18,20}$ observed at Nieuwkoop and Faber stage 27 (Figure 1A). At stage 32, the presumptive lens is polarized with anterior cells differing in their aspect from posterior cells, and hence is now referred to as a lens vesicle (Figure 1B). At these stages, the apparent detachment of the lens rudiment or vesicle from the sensory ectoderm (Figures 1A-B) is probably artifactual.

In mammals, the posterior cells of the lens vesicle elongate to form primary lens fiber cells, while the anterior cells form an epithelial layer ${ }^{1,26-28}$. This is comparable to Xenopus stage 38, when a layer of epithelial cells surrounds the primary fiber cells (Figure 1C). The primary fiber cells were reported to form between stages 35 and $41^{18}$. The primary lens fiber cells then progressively lose their nuclei and other organelles. Kariolysis is detected at Xenopus stage 41 (Figure 1D). The high magnification of stage 41 lens reveals the absence of nuclei in the centralmost fiber cells (Figure 1D').

In mammals, the anterior epithelium is the place of cell divisions. In the so-called transition zone in the equatorial region of the lens, epithelial cells exit the cell cycle, and initiate differentiation into secondary fiber cells which internalize. These secondary fiber cells progressively form rings around the primary fiber cells. This results in a lens nucleus made of the original primary fiber cells and a lens cortex made of the secondary fiber cells. The gradual loss of organelles and nuclei in the fiber cells, and their elongation along the antero-posterior axis curresponding to the light path, support lens transparency and light transmission toward the retina 1,26-28. This is essentially the same situation in stage 44 Xenopus larvae. However, while in mammals the epithelium is essentially anterior, it seems to cover the fiber cells both anteriorly and posteriorly in Xenopus (Figure 1E). At stage 47, only the epithelial cells that surround the lens have retained their nuclei. The inner fibers are oriented around the antero-posterior axis and the lens is in the form of many concentric layers (Figure 1F, F'). At stage 48 (Figure 1G), the lens has grown further and has the same structure as in adults ${ }^{29}$. 


\section{Strong alterations of eye development in pax6 crispants}

pax6 is a key transcription factor regulator gene in eye development ${ }^{30-33}$, and TALENmediated inactivation of pax6 gene has been described both in X. laevis and X. tropicalis ${ }^{34,35}$. To demonstrate that eye phenotypes can readily be observed following CRISPR/Cas9 mediated gene engineering, we co-injected Cas9 nuclease with a single sgRNA against pax6 exon 5 in X. laevis embryos soon after fertilization. $X$. laevis is allotetraploid, and homoeologous genes (pairs of homologous genes found together in the same allopolyploid genome) are distinguished by their suffix ".L" and ".S" ${ }^{36}$. The region targeted by the pax6 sgRNA is fully conserved between pax6.L and pax6.S. We amplified and sequenced the targeted loci with homoeologous-specific primers. Figure 2A shows the Sanger chromatograms obtained by sequencing the pax6.L and pax6.S loci amplified from one control and one sgRNA and Cas9-injected embryo. In the edited tadpole, several nucleotides are overlaid at each position around the cleavage site, revealing its mosaicism. We used the ICE (Inference of CRISPR Edits) software to infer the composition of this mixture of sequences 37. For each chromatogram, ICE returns a percentage of sequences with insertions-deletions (InDels), which is a proxy for the efficiency of genome editing. ICE analysis revelead genome editing efficiencies in several embryos to be close to 100\%, both for pax6.L and pax6.S (Figure 2B).

As compared with control larvae (Figure 2C, upper panel), disrupting pax6 does not strongly modify the overall morphology of stage 41-42 larvae (Figures 2D-E, upper panels). However, eye morphologies of pax6 crispants are affected in the injected embryos. The affected eyes have different aspects, as shown in Figures 2D-E, middle panels. Overall, the number of tadpoles with defective eye morphology is close to 95\% (Figure 2F). Sections reveal no lens and a highly usorganised and/or hypotrophied retina (Figures 2D-E, lower panels). To confirm these data, we designed another sgRNA against pax6 exon 5. Gene inactivation mediated by this second sgRNA leads to externally visible eye defects in $84 \%$ of the injected tadpoles (Figure 2F), confirming gene editing specificity.

We expected this abnormal eye structure to lead to blindness. To test this hypothesis, we set up a functional vision assay in Xenopus larvae. This assay relies on the preference of tadpoles for white when placed in a tank with both white and black sides ${ }^{38}$. Within a pool of 10 tadpoles, we 
counted the number on the white side every minute for 10 minutes. We repeated this experiment for 8 independent pools. While the cumulative number of tadpoles on white would be close to 50 (corresponding to $50 \%$ of the time spent on white) if the tadpoles had no preference for the white side, we observed that control tadpoles spend around $80 \%$ of their time on the white side (95\% confidence interval $[67 ; 86])$. pax6 crispants spend almost the same time on either the white or black background (95\% confidence interval of the percentage of time spent on white [50; 59]) (Figure 2G). This strongly suggests that pax6 crispants are virtually blind, though a defective development of the central nervous system in embryos lacking Pax6 ${ }^{39}$ may be an alternative explanation to these observations.

\section{Disruption of gja8 causes a strong cataract}

In human, GJA8 encodes connexin 50, a transmembrane gap junction protein which supports the diffusion of small molecules between neighbouring lens fibers. Genetic variations of GJA8 cause congenital cataracts ${ }^{40,41}$, but can also be involved in age-related cataracts ${ }^{42}$.

We tested if inactivating gja8 in $X$. laevis could model human cataracts by co-injecting Cas9 nuclease and a sgRNA directed against gja8 exon 1. This sgRNA is expected to target both gja8 homoeologous genes. We separately sequenced gja8.L and gja8.S loci after homoeologous locusspecific amplification to assess gene targeting efficiency. Sanger chromatograms confirmed the mosaicism of gja8 crispants (Figure 3A). ICE analysis revealed that gja8 crispants contain around 80\% InDels at gja8.L and gja8.S loci (Figure 3B). To confirm the ICE data, we subcloned one gja8.L amplimere and we sequenced 13 subclones. The obtained sequences and their number of uccurences are consistent with the ICE results (Figure 3C). Together, these data show that the gja8 sgRNA targets both gja8.L and gja8.S with a good efficiency.

The overall morphology of gja8 crispants is completely unaltered, compared with sibling buffer-injected embryos (Figures 3D, E, upper panels). However, the eyes of gja8 crispants have greyish appearance in the central region, which is not observed in control embryos (middle panels). This is highly reminiscent of cataract, where lens clouding impedes light diffusion and correct observation of the dark pigmented retinal epithelium. A large proportion (78\%, 213/278) of gja8 
crispants have such a cataract detected under a simple binocular magnifier (Figure 3F). Phenotype specificity was confirmed by injecting another sgRNA against gja8, which leads to a similar cataract phenotype albeit with a reduced efficiency (Figure 3F).

Histological sections revealed that the morphology of the eye is essentially unaffected in gja8 crispants (Figure 3D-E, lower panels). The retina has all the cell layers found in control eyes. However, the lenses are smaller in crispants, and the inner cells of crispant lenses retain their nuclei, demonstrating a defective karyolysis that should have occurred at this stage. DAPI staining of lens sections confirm nuclei retention in gja8 crispant lenses (Figures 3G-H). Phalloidin staining reveals that the actin fibers are aligned along the antero-posterior axis in control lenses (Figure 3G). In mammals, this organisation supports the elongation of lens fiber cells along the light path. Interestingly, it appears abnormal in gja8 crispant lenses (Figure $3 \mathrm{H}$ ). Hence, at least two features that challenge lens transparency provide a potential explanation for the development of cataract in gja8 crispants, namely, defective organisation of fiber cells along the antero-posterior axis and the abnormal retention of their nuclei in the central region of the lens. Mice or rabbits disrupted for Gja8 show a reduced size of the lens, an arrest of the differentiation of lens fiber cells, and a retention of nuclei ${ }^{43-45}$. Hence, our observations in Xenopus recapitulate the phenotypes observed in mammals.

We next asked if lens clouding affects the vision of the tadpoles. In the same functional vision assay as before, gja8 crispants have a preference for the white background, indicating that they are not blind (95\% confidence interval of the percentage of time spent on the white side of the tank [58; 65]). However, the crispant embryos spend much less time on white than sibling control embryos (Figure 3I. $\mathrm{p}=9.1 \times 10^{-4}$, Wilcoxon test). Hence, the cataract caused by disrupting the gja8 gene reduces but does not abrogate the visual acuity of Xenopus tadpoles.

\section{Weak cataract in dnase $2 b$ crispants}

In mice, Dnase2b encodes a nuclease responsible of degrading DNA during lens fiber differentiation, and Dnase $2 b$-KO mice develop cataract associated with nuclei retention ${ }^{46}$. We asked therefore if inactivating dnase $2 b$ in Xenopus also elicits cataract in tadpoles. While dnase2b.S 
gene is annotated in Xenopus laevis 9.1 genome, it is not the case of dnase2b.L. BLAST search identified putative dnase2b.L exons 1 and 2, but the other exons are apparently absent. Hence, there is probably only one functional dnase $2 b$ homoeologous gene, dnase2b.S, while dnase2b.L may have evolved as a pseudogene. Nevertheless, we co-injected Cas9 enzyme with a mixture of sgRNA against the first exons of dnase2b.S gene and dnase2b.L possible pseudogene. As we failed to amplify this putative dnase2b.L exon 1 , we only assessed the efficiency of dnase $2 b . S$ gene editing by Sanger sequencing (Figure 4A) and ICE analysis. Gene editing was moderately efficient, with roughly $50 \%$ of InDels (Figure 4B). We found no evidence for lens clouding comparable to what had been observed in gja8 crispants, and most larvae have no eye defect detectable by external observation. However, one fifth of the larvae (46/233) have a smaller eye, with a gap in the ventral part of the retina suggesting chorioretinal coloboma (Figures 4C-D). We assessed the vision of dnase $2 b$ crispants. They tend to avoid the dark side of the tank, demonstrating that they are able to distinguish white from black (95\% confidence interval of the percentage of time spent on the white side of the tank [61; 71]). However, compared with control embryos, their preference for white is

reduced (Figure 4E. $\mathrm{p}=3.7 \times 10^{-3}$, Wilcoxon test). We conclude from these observations that dnase $2 b$ crispants have poorer visual acuity, and microphthalmia and possibly coloboma albeit not in a fully penetrant manner.

We made a series of sections to understand the anatomical bases of reduced visual acuity. At stage 41 the posterior poles of both control and dnase $2 b$ crispant lenses are made of a layer of several nucleated epithelial cells. However, while this layer corresponds to almost half of the dnase2b crispant lenses, it is much thinner in control lenses (Figures 4F-G). The posterior layer of nucleated cells is also thicker in dnase $2 b$ crispant lenses at stage 45 (Figures $4 \mathrm{H}-\mathrm{I}$ ). The most likely explanation for these observations is that, while lens fiber differentiation in control lenses is accompanied by a progressive loss of nuclei, the kariolysis is delayed in dnase $b$ crispant lenses, resulting in an increased number of nucleated cells within the lens.

\section{Toward screening anti-cataract compounds in Xenopus tadpoles}

The above results show that cataract can be elicited in Xenopus tadpoles by genome engineering, and we tested if this model responds to potential anti-cataract compounds. The 
chemical chaperone 4-phenylbutyrate (4PBA) mitigates cellular defects associated with a GJA8 mutation (Cx50D47N) in HeLa cells and in a mouse model. However, it does not reduce lens opacification ${ }^{47}$. We tested if this compound has a detectable effect on gja8 crispant tadpoles. In preliminary experiments, we found that a dose of $0.025 \mathrm{mM}$ 4PBA produces no morphological defect and is apparently not toxic. This is consistent with a previous report in zebrafish where 4PBA was used in a model of dominant osteogenesis imperfecta ${ }^{48}$. Indeed, in vision assays, treated and untreated control embryos behave identically (Figure 5, 2 left panels). We treated gja8 crispants with 4 PBA for $48 \mathrm{~h}$. We found no difference between treated and untreated tadpoles regarding lens clouding in external examination. However, we observed a tendency toward a vision improvement upon 4PBA treatment ( $\mathrm{p}=$ 0.067, Figure 5, 2 right panels). Hence, 4PBA, which showed a limited capacity to improve pathological issues caused by a GJA8 mutation in HeLa cells and mice, apparently has the same effect in Xenopus gja8 crispants. This suggests that cataract-prone Xenopus tadpoles can be used to test the efficiency of potential anti-cataract drugs.

\section{DISCUSSION}

We disrupted three genes in Xenopus laevis by CRISPR/Cas9, and we investigated the resulting eye phenotypes after a few days of development. pax6 crispants have highly severe eye phenotypes, consistent with previous reports ${ }^{34,35}$. gja8 crispants have lens-specific defects, with clouded lenses directly visible by external observations, and defective karyolysis and organisation of fiber cells in sections. Finally, dnase $2 b$ crispants have a wild-type appearance in external observation and a thickened layer of posterior epithelial cells in sections, suggesting a blockage of lens fiber cells differentiation. Interestingly, functional vision assays reflect this graduation. This suggests that these vision assays can prove useful to quantitatively assess cataract severity, for instance in a process of screening potential anti-cataract drugs. Because the molecular chaperone 4PBA was reported to have a limited capacity to mitigate some cellular defects associated with a

mutation in $G J A 8{ }^{47}$, we tested the same molecule on gja8 crispants. We found that 4PBA may have a weak capacity to improve the vision of gja8 crispants. The weakness of 4PBA may be due to the differences in the nature of the GJA8 mutations between our study and the previous study (i.e. Cx50D47A). In any case, this data reinforces the notion that functional vision assays in Xenopus cataract-prone tadpoles are a novel and relevant tool in a process of anti-cataract drug development. 
Indeed, no pharmacological treatment of cataract currently exists, but recent findings indicate that sterol-based compounds hold promise to prevent/treat cataracts ${ }^{49,50}$. These studies report that steroid compounds reduce the aggregation of mutant crystallin protein forming amyloid fibrils in vitro and in cultured cells, and reduce cataract severity in rabbit or mouse models. Other compounds could certainly be screened in preclinical approaches to isolate novel molecules with a potential to enter clinical trials against cataracts. A promising tool toward this goal is the "micro-lens" obtained in vitro by differentiation of human stem cells ${ }^{9}$. However, this organoid does not recapitulate the normal environment or the barriers of the eye (especially the cornea) that may prevent molecules of potential pharmaceutical interest to access to the lens. Furthermore, obtaining a large number of genetically modified Xenopus tadpoles is cheap and rapid, and larvae are generally not submitted to regulations on animal tests. For these reasons, we think that the genetically modified cataract-prone Xenopus tadpoles that we describe here are a promising novel preclinical model to screen potential anti-cataract drugs.

Having shown that cataract can be readily observed in Xenopus will also permit to tackle a subset of poorly understood congenital genetic cataracts. Tens of genes were identified by genetic approaches in human as involved in congenital genetic cataract. Most of these genes encode crystallins, the major proteins of the lens that make a structure for refraction of light, connexins (like that encoded by the GJA8 gene), which exchange small molecules between cells, proteins of the cell membrane, the extracellular matrix or the cytoskeleton that set up the highly peculiar morphology of lens fibres, or transcription factors such as FOXE3, HSF4, MAF, and PITX3 ${ }^{2,5,51 .}$ However, for some other genes, the link between the molecular functions of the encoded proteins and cataract remains elusive. Understanding the involvement of these genes in cataract can be achieved by constructing and characterizing new animal models inactivated for these genes. Indeed, the bioinformatics resource tool iSyTE has predicted several promising candidates that need to be validated in animal models ${ }^{8,16,51-55}$. The cost-effective pipeline of cataract investigation in Xenopus that we describe here would help to uncover the genetic basis of these rare genetic cataracts as well as to identify/validate new cataract-linked genes. 


\section{EXPERIMENTAL PROCEDURES}

\section{Ethics statement}

The animals were housed in the Xenopus facilities of the IGDR as approved by the French animal care agency (Direction des Services Vétérinaires). Experiments were carried out according to standard procedures following local ethics committee opinion and acceptance by the ministry of research (APAFIS 14829, 2018).

\section{Gene editing in Xenopus laevis}

CRISPR/Cas9-mediated gene editing in Xenopus laevis was essentially as described for Xenopus tropicalis ${ }^{56}$. We designed the sgRNAs (single guide RNA) with CHOPCHOP ${ }^{57}$. We prepared sgRNAs by in vitro transcription from templates obtained by PCR with a 3' primer common to all sgRNA templates ${ }^{56}$ and the following 5 ' primers:

pax6, sgRNA \#1 (Targetting exon5)

TAATACGACTCACTATAGGAGACCGGATCGATCCGACCTGTTTTAGAGCTAGAAATAGCA pax6, sgRNA \#2 (Targetting exon5)

TAATACGACTCACTATAGGATCCGACCTCGGGCGATCGGGTTTTAGAGCTAGAAATAGCA gja8, sgRNA \#1 (Targetting an exon annotated as exon 1 in gja8.L and exon 2 in gja8.S) TAATACGACTCACTATAGGTTTGAGGTGGGCTTTGTGGTGTTTTAGAGCTAGAAATAGCA gja8, sgRNA \#2 (Targetting an exon annotated as exon 1 in gja8.L and exon 2 in gja8.S) TAATACGACTCACTATAGGCTCTCTCACACATTAGGCTGGTTTTAGAGCTAGAAATAGCA dnase $2 b . S$ exon

TAATACGACTCACTATAGGTGGGGTCACCAGCTTCATTTGTTTTAGAGCTAGAAATAGCA dnase2b.L (Targetting exon1)

TAATACGACTCACTATAGGATTTCAGCATGAAATCTCAGGTTTTAGAGCTAGAAATAGCA

The gene-specific sequences are in bold underlined and are flanked by the $\mathrm{T} 7$ promoter and a region hybridizing to the 3 ' primer.

We dejellyed the embryos 15 minutes after fertilization, and we injected them up to 45 minutes after fertilization with $9.2 \mathrm{nl}$ of a mixture of Cas9 enzyme (IDT DNA 1085059, $0.5 \mu \mathrm{g} / \mu \mathrm{l}$ ) and sgRNA $(0.2 \mu \mathrm{g} / \mu \mathrm{l})$ in $12 \mathrm{mM}$ HEPES (pH 7.5), $90 \mathrm{mM} \mathrm{KCl}$. We allowed them to develop at $22^{\circ} \mathrm{C}$ following standard procedures and we staged them according to Nieuwkoop and Faber ${ }^{58}$. 
We checked gene editing by lysing the embryos and sequencing the PCR products obtained with the following primers:

pax6.S TCAGTTCTGCGACAGAGTAGGC and AATAGCACTCACTTACACTGGGG (amplification), sequencing with the forward primer;

pax6.L GACATGTAAGGGGCTATGTGC and GGGATATTGTCGTTGGTACAGA (amplification), TTAATGCTACCTATAAACTA (sequencing);

gja8.L (sgRNA \#1) ATGAGCACTCGACTGTGATCG and GTTTGGACAGGGCCACCTAC (amplification), sequencing with the forward primer;

gja8.S (sgRNA \#1) TTTTGTGTGCAACACTCAGCAG and GGATGCTGATCTTCGCTCCTCC (amplification), sequencing with the forward primer;

gja8.L (sgRNA \#2) GATCGGTAGAGTTTGGCTCACT and GTTTGGACAGGGCCACCTAC (amplification), sequencing with the forward primer;

gja8.S (sgRNA \#2) GATCGGTAGAGTTTGGCTCACT and GGATGCTGATCTTCGCTCCTCC (amplification), sequencing with the forward primer;

dnase2b.S TCTGACAGAAAACCGTGCCA and AGCGTTTGTGTTCTCCCCTT (amplification), sequencing with the forward primer.

The traces (chromatograms) were uploaded on the ICE server (https://ice.synthego.com/\#/) and ICE analysed with the default parameters.

\section{Histological methods}

For histological analyses, we fixed and embedded in paraffin wax embryos at the indicated stages. Horizontal sections were stained with Hematoxylin and Eosin. For histochemical analyses, we fixed embryos in 4\% PFA in PBS for $1 \mathrm{~h}$, rinsed them in PBS and incubated them in $30 \%$ sucrose uvernight at $4^{\circ} \mathrm{C}$. Embryos were embedded in Tissue Teck and stored at $-80^{\circ} \mathrm{C}$. Transversal cryosections were performed and stained with Phalloidin and DAPI.

\section{Functional vision assays}

The functional vision assays were essentially as described ${ }^{38}$. We put independent pools of 10 embryos in a rectangular water tank with a black and a white side. We switched the sides and we counted the numbers of embryos on the white side one minute after switching the sides. We repeated this operation every minute for 10 minutes. The cumulative number of embryos on the 
white is comprised between 0 and 100 and corresponds to the percentage of time spent on white. We show it for 8-10 independent pools of tadpoles. We assessed the significance of the results with $\mathrm{R}$, including the CI function in Rmisc (https://cran.r-project.org/package=Rmisc). Functional vision assays of 4PBA-treated tadpoles were carryed out on gja8 crispants and controls incubated for 48h, starting at stage 44, in $0.025 \mathrm{mM} 4 \mathrm{PBA}$ in water (Sigma P21006) and allowed to recover overnight in pure water.

\section{ACKNOWLEDGMENTS}

We thank the Biosit platforms (Rennes, France) for access to their facilities, and notably MRic (microscopy) and H2P2 (histology).

\section{REFERENCES}

1. Cvekl A, Zhang X. Signaling and Gene Regulatory Networks in Mammalian Lens Development. Trends Genet. 2017;33(10):677-702. doi:10.1016/j.tig.2017.08.001

2. Shiels A, Hejtmancik JF. Mutations and mechanisms in congenital and age-related cataracts. Exp Eye Res. 2017;156:95-102. doi:10.1016/j.exer.2016.06.011

3. Foster A, Gilbert C. Cataract in children. Acta Paediatr. 2003;92(12):1376-1378.

4. Francis PJ, Berry V, Bhattacharya SS, Moore AT. The genetics of childhood cataract. J Med Genet. 2000;37(7):481-488.

5. Shiels A, Hejtmancik JF. Molecular Genetics of Cataract. Prog Mol Biol Transl Sci. 2015;134:203-218. doi:10.1016/bs.pmbts.2015.05.004

6. Nibourg LM, Gelens E, Kuijer R, Hooymans JMM, van Kooten TG, Koopmans SA. Prevention of posterior capsular opacification. Exp Eye Res. 2015;136:100-115. doi:10.1016/j.exer.2015.03.011

7. Wormstone IM, Wang L, Liu CSC. Posterior capsule opacification. Exp Eye Res. 2009;88(2):257-269. doi:10.1016/j.exer.2008.10.016 
8. Anand D, Lachke SA. Systems biology of lens development: A paradigm for disease gene discovery in the eye. Exp Eye Res. 2017;156:22-33. doi:10.1016/j.exer.2016.03.010

9. Murphy P, Kabir MH, Srivastava T, et al. Light-focusing human micro-lenses generated from pluripotent stem cells model lens development and drug-induced cataract in vitro. Development. 2018;145(1). doi:10.1242/dev.155838

10. Goishi K, Shimizu A, Najarro G, et al. AlphaA-crystallin expression prevents gamma-crystallin insolubility and cataract formation in the zebrafish cloche mutant lens. Development. 2006;133(13):2585-2593. doi:10.1242/dev.02424

11. Chan C-C, ed. Animal Models of Ophthalmic Diseases. Springer International Publishing; 2016. https://www.springer.com/fr/book/9783319194332. Accessed April 11, 2019.

12. Huynh M-H, Zhu SJ, Kollara A, Brown T, Winklbauer R, Ringuette M. Knockdown of SPARC leads to decreased cell-cell adhesion and lens cataracts during post-gastrula development in Xenopus laevis. Dev Genes Evol. 2011;220(11-12):315-327. doi:10.1007/s00427-010-0349-x

13. Maher GJ, Hilton EN, Urquhart JE, et al. The cataract-associated protein TMEM114, and TMEM235, are glycosylated transmembrane proteins that are distinct from claudin family members. FEBS Lett. 2011;585(14):2187-2192. doi:10.1016/j.febslet.2011.05.060

14. Pfirrmann T, Emmerich D, Ruokonen P, et al. Molecular mechanism of CHRDL1-mediated Xlinked megalocornea in humans and in Xenopus model. Hum Mol Genet. 2015;24(11):31193132. doi:10.1093/hmg/ddv063

15. Rothe M, Kanwal N, Dietmann P, et al. An Epha4/Sipa1l3/Wnt pathway regulates eye development and lens maturation. Development. 2017;144(2):321-333. doi:10.1242/dev.147462

16. Siddam AD, Gautier-Courteille C, Perez-Campos L, et al. The RNA-binding protein Celf1 posttranscriptionally regulates p27Kip1 and Dnase2b to control fiber cell nuclear degradation in lens development. PLoS Genet. 2018;14(3):e1007278. doi:10.1371/journal.pgen.1007278

17. McDevitt DS, Brahma SK. Ontogeny and localization of the crystallins during embryonic lens development in Xenopus laevis. J Exp Zool. 1973;186(2):127-140. doi:10.1002/jez.1401860204 
18. Brunekreef GA, van Genesen ST, Destrée OH, Lubsen NH. Extralenticular expression of Xenopus laevis alpha-, beta-, and gamma-crystallin genes. Invest Ophthalmol Vis Sci. 1997;38(13):2764-2771.

19. Ishibashi S, Yasuda K. Distinct roles of maf genes during Xenopus lens development. Mech Dev. 2001;101(1-2):155-166. doi:10.1016/s0925-4773(00)00585-2

20. Mizuno N, Mochii M, Takahashi TC, Eguchi G, Okada TS. Lens regeneration in Xenopus is not a mere repeat of lens development, with respect to crystallin gene expression. Differentiation. 1999;64(3):143-149. doi:10.1046/j.1432-0436.1999.6430143.x

21. Henry JJ, Elkins MB. Cornea-lens transdifferentiation in the anuran, Xenopus tropicalis. Dev Genes Evol. 2001;211(8-9):377-387. doi:10.1007/s004270100163

22. Henry JJ, Tsonis PA. Molecular and cellular aspects of amphibian lens regeneration. Prog Retin Eye Res. 2010;29(6):543-555. doi:10.1016/j.preteyeres.2010.07.002

23. Servetnick MD, Cook TL, Grainger RM. Lens induction in axolotls: comparison with inductive signaling mechanisms in Xenopus laevis. Int J Dev Biol. 1996;40(4):755-761.

24. Vergara MN, Tsissios G, Del Rio-Tsonis K. Lens regeneration: a historical perspective. Int $J$ Dev Biol. 2018;62(6-7-8):351-361. doi:10.1387/ijdb.180084nv

25. Malloch EL, Perry KJ, Fukui L, et al. Gene Expression Profiles of Lens Regeneration and Development in Xenopus laevis. Dev Dyn. 2009;238(9):2340-2356. doi:10.1002/dvdy.21998

Ogino H, Ochi H, Reza HM, Yasuda K. Transcription factors involved in lens development from the preplacodal ectoderm. Dev Biol. 2012;363(2):333-347. doi:10.1016/j.ydbio.2012.01.006

27. Rao PV, Maddala R. The role of the lens actin cytoskeleton in fiber cell elongation and differentiation. Semin Cell Dev Biol. 2006;17(6):698-711. doi:10.1016/j.semcdb.2006.10.011

28. Wride MA. Lens fibre cell differentiation and organelle loss: many paths lead to clarity. Philos Trans R Soc Lond, B, Biol Sci. 2011;366(1568):1219-1233. doi:10.1098/rstb.2010.0324 
29. Wiechmann AF, Wirsig-Wiechmann CR. Color Atlas of Xenopus Laevis Histology. Springer US; 2003. https://www.springer.com/us/book/9781461348764. Accessed April 11, 2019.

30. Altmann CR, Chow RL, Lang RA, Hemmati-Brivanlou A. Lens induction by Pax-6 in Xenopus laevis. Dev Biol. 1997;185(1):119-123. doi:10.1006/dbio.1997.8573

31. Chow RL, Altmann CR, Lang RA, Hemmati-Brivanlou A. Pax6 induces ectopic eyes in a vertebrate. Development. 1999;126(19):4213-4222.

32. Halder G, Callaerts P, Gehring WJ. Induction of ectopic eyes by targeted expression of the eyeless gene in Drosophila. Science. 1995;267(5205):1788-1792.

33. Onuma Y, Takahashi S, Asashima M, Kurata S, Gehring WJ. Conservation of Pax 6 function and upstream activation by Notch signaling in eye development of frogs and flies. Proc Natl Acad Sci USA. 2002;99(4):2020-2025. doi:10.1073/pnas.022626999

34. Nakayama T, Fisher M, Nakajima K, et al. Xenopus pax6 mutants affect eye development and other organ systems, and have phenotypic similarities to human aniridia patients. Dev Biol. 2015;408(2):328-344. doi:10.1016/j.ydbio.2015.02.012

35. Suzuki K-IT, Isoyama Y, Kashiwagi K, et al. High efficiency TALENs enable F0 functional analysis by targeted gene disruption in Xenopus laevis embryos. Biol Open. 2013;2(5):448-452. doi:10.1242/bio.20133855

36. Session AM, Uno Y, Kwon T, et al. Genome evolution in the allotetraploid frog Xenopus laevis. Nature. 2016;538(7625):336-343. doi:10.1038/nature19840

37. Hsiau T, Maures T, Waite K, et al. Inference of CRISPR Edits from Sanger Trace Data. bioRxiv. January 2018:251082. doi:10.1101/251082

38. Viczian AS, Zuber ME. A simple behavioral assay for testing visual function in Xenopus laevis. J Vis Exp. 2014;(88). doi:10.3791/51726 
39. Huettl R-E, Eckstein S, Stahl T, et al. Functional dissection of the Pax6 paired domain: Roles in neural tube patterning and peripheral nervous system development. Dev Biol. 2016;413(1):86103. doi:10.1016/j.ydbio.2015.07.009

40. Shiels A, Mackay D, Ionides A, Berry V, Moore A, Bhattacharya S. A missense mutation in the human connexin50 gene (GJA8) underlies autosomal dominant “zonular pulverulent” cataract, on chromosome 1q. Am J Hum Genet. 1998;62(3):526-532. doi:10.1086/301762

41. Yi J, Yun J, Li Z-K, Xu C-T, Pan B-R. Epidemiology and molecular genetics of congenital cataracts. Int J Ophthalmol. 2011;4(4):422-432. doi:10.3980/j.issn.2222-3959.2011.04.20

42. Yu X, Ping X, Zhang X, et al. The impact of GJA8 SNPs on susceptibility to age-related cataract. Hum Genet. 2018;137(11-12):897-904. doi:10.1007/s00439-018-1945-5

43. Rong P, Wang X, Niesman I, et al. Disruption of Gja8 (alpha8 connexin) in mice leads to microphthalmia associated with retardation of lens growth and lens fiber maturation. Development. 2002;129(1):167-174.

44. White TW, Goodenough DA, Paul DL. Targeted ablation of connexin50 in mice results in microphthalmia and zonular pulverulent cataracts. J Cell Biol. 1998;143(3):815-825.

45. Yuan L, Sui T, Chen M, et al. CRISPR/Cas9-mediated GJA8 knockout in rabbits recapitulates human congenital cataracts. Sci Rep. 2016;6:22024. doi:10.1038/srep22024

46. Nishimoto S, Kawane K, Watanabe-Fukunaga R, et al. Nuclear cataract caused by a lack of DNA degradation in the mouse eye lens. Nature. 2003;424(6952):1071-1074. doi:10.1038/nature01895

47. Jara O, Minogue PJ, Berthoud VM, Beyer EC. Chemical chaperone treatment improves levels and distributions of connexins in Cx50D47A mouse lenses. Exp Eye Res. 2018;175:192-198. doi:10.1016/j.exer.2018.06.015

48. Gioia R, Tonelli F, Ceppi I, et al. The chaperone activity of 4PBA ameliorates the skeletal phenotype of Chihuahua, a zebrafish model for dominant osteogenesis imperfecta. Hum Mol Genet. 2017;26(15):2897-2911. doi:10.1093/hmg/ddx171 
49. Makley LN, McMenimen KA, DeVree BT, et al. Pharmacological chaperone for $\alpha$-crystallin partially restores transparency in cataract models. Science. 2015;350(6261):674-677. doi:10.1126/science.aac9145

50. Zhao L, Chen X-J, Zhu J, et al. Lanosterol reverses protein aggregation in cataracts. Nature. 2015;523(7562):607-611. doi:10.1038/nature14650

51. Anand D, Kakrana A, Siddam AD, Huang H, Saadi I, Lachke SA. RNA sequencing-based transcriptomic profiles of embryonic lens development for cataract gene discovery. Hum Genet. 2018;137(11-12):941-954. doi:10.1007/s00439-018-1958-0

52. Kakrana A, Yang A, Anand D, et al. iSyTE 2.0: a database for expression-based gene discovery in the eye. Nucleic Acids Res. 2018;46(D1):D875-D885. doi:10.1093/nar/gkx837

53. Lachke SA, Ho JWK, Kryukov GV, et al. iSyTE: integrated Systems Tool for Eye gene discovery. Invest Ophthalmol Vis Sci. 2012;53(3):1617-1627. doi:10.1167/iovs.11-8839

54. Aryal S, Anand D, Hernandez FG, et al. MS/MS in silico subtraction-based proteomic profiling as an approach to facilitate disease gene discovery: application to lens development and cataract. Hum Genet. December 2019. doi:10.1007/s00439-019-02095-5

55. Dash S, Brastrom LK, Patel SD, Scott CA, Slusarski DC, Lachke SA. The master transcription factor SOX2, mutated in anophthalmia/microphthalmia, is post-transcriptionally regulated by the conserved RNA-binding protein RBM24 in vertebrate eye development. Hum Mol Genet. December 2019. doi:10.1093/hmg/ddz278

๖ь. Nakayama T, Blitz IL, Fish MB, et al. Cas9-based genome editing in Xenopus tropicalis. Meth Enzymol. 2014;546:355-375. doi:10.1016/B978-0-12-801185-0.00017-9

57. Labun K, Montague TG, Gagnon JA, Thyme SB, Valen E. CHOPCHOP v2: a web tool for the next generation of CRISPR genome engineering. Nucleic Acids Res. 2016;44(W1):W272-276. doi:10.1093/nar/gkw398 
58. Nieuwkoop PD, Faber J, eds. Normal Table of Xenopus Laevis (Daudin): A Systematical and Chronological Survey of the Development from the Fertilized Egg till the End of Metamorphosis. New York:

Garland

Pub; 1994. 


\section{FIGURE LEGENDS}

\section{Figure 1. Eye development in Xenopus laevis.}

Representative sections of embryos staged according to Nieuwkoop and Faber ${ }^{58}$, hematoxylineeosine stained. A, Stage 27, 30 hours post-fertilization at $23^{\circ} \mathrm{C}$. Ir lens rudiment; oc optic cup. B, Stage 32, 40 hours post-fertilization. lv lens vesicle. C, Stage 38, 2 days 5 hours post-fertilization. le lens epithelium; plfc primary lens fiber cells; rpe retinal pigmented epithelium. D, Stage 41, 3 days 4 hours post-fertilization. D', Higher magnification of the lens shown in $\mathbf{D}$, with interpretative diagram. E, Stage 44, 3 days 20 hours post-fertilization. lfc, lens fiber cells. F, Stage 47, 5.5 days post-fertilization. gcl, ganglion cell layer; inl, inner nuclear layer; oc, outer cornea; onl, outer nuclear layer; pr, photoreceptors; rpe retinal pigmented epithelium. F', Higher magnification of the lens shown in F. G, Stage 48, 1 week post-fertilization. All scale bars $200 \mu \mathrm{m}$ except F' $90 \mu \mathrm{m}$.

\section{Figure 2. Eye phenotype of pax6 crispants.}

We injected embryos with Cas9 enzyme and a sgRNA targeted against pax6.L and pax6.S or buffer, and we allowed the embryos to develop until stage 41-42. A, We separately amplified the pax6.L and pax6.S loci from total embryos for Sanger sequencing. We show representative chromatograms of one sgRNA and one buffer-injected embryo. The sgRNA and PAM sequences are underlined and the cleavage sites are shown. B, The percentages of InDels in individual embryos were calculated from Sanger chromatograms with ICE ${ }^{37}$. C-E, from top to bottom whole embryo, higher magnification of the eye and histological section of the eye, scale bar $200 \mu \mathrm{m}$. C, Buffer-injected embryo. D-E, Two Cas9 and sgRNA injected embryos. Key for lens and retina layers, see Figure 1. F, Quantification of eye defects, in tadpoles previously injected with the same sgRNA against pax6 as above (sgRNA \#1) or an alternative sgRNA against pax6 (sgRNA \#2) G, Functional vision assay un stage 45 buffer-injected embryos and pax6 crispants (sgRNA \#1). We put 10 tadpoles in a water tank with a black and a white side, we switched the sides and we counted the number of embryos on the white side after one minute. We repeated the switching-counting procedure 10 times for each batch of 10 tadpoles. The cumulative number of embryos on white (between 0 and 100) is shown here, for 8 independent pools of 10 tadpoles. The tadpoles spend more time on white if they are able to distinguish white from black, and the bias in favour of the white side reflects their visual acuity.

\section{Figure 3. Eye phenotype of gja8 crispants.}


We injected embryos with Cas9 enzyme and a sgRNA targeted against gja8.L and gja8.S, and we allowed the embryos to develop until stage 47. A-B, Representative Sanger chromatograms of one sgRNA and one buffer-injected embryo and percentages of InDels in individual embryos, as in Figures 2A-B. C, We subcloned the gja8.L amplimere from one gja8 crispant and we sequenced individual clones. We show the sequences and their number of occurences. ICE is the result of the ICE analysis carried out on the same embryo. D-E, from top to bottom whole embryo, higher magnification of the eye, and histological section of the eye, scale bar $200 \mu \mathrm{m}$. D, Buffer-injected embryo. E, Cas9 and sgRNA-injected embryo. Note the greyish lens. F, Quantification of cataract, in tadpoles previously injected with the same sgRNA against gja8as above (sgRNA \#1) or an alternative sgRNA against gja8 (sgRNA \#2) G-H, From top to bottom, DAPI staining, Phalloidin staining, and Merge of lens sections. A and P highlight the anterior and posterior pole of the lens. G, Buffer-injected embryo. H, Cas9 and sgRNA \#1-injected embryo. I, Functional vision assay of stage 47 buffer-injected embryos and gja8 crispants (sgRNA \#1), as in Figure 2G.

\section{Figure 4. Eye phenotype of dnase $2 b$ crispants.}

We injected embryos with Cas9 enzyme and a mixture of sgRNA targeted against dnase2b.S and a putative dnase2b.L locus, and we allowed the embryos to develop. A-B, Representative Sanger chromatograms of one sgRNA and one buffer-injected embryo and percentages of InDels in individual embryos, as in Figures 2A-B. Since we failed to amplify the putative dnase2b.L locus, only the results for dnase2b.S are shown. C-D, Stage 41 larvae previously injected with buffer (C) or Cas9-sgRNA (D). Insets are high magnifications of the eyes of the same larvae. E, Functional vision assay of buffer-injected embryos and dnase $2 b$ crispants, as in Figure 2F. F, Histological section of the eye of a buffer-injected stage 41 tadpole. G, Section of the eye of a Cas9 and sgRNAinjected stage 41 tadpole with microphthalia. H-I, Same as F-G with stage 45 tadpoles. Scale bars $200 \mu \mathrm{m}$.

\section{Figure 5. Effect of 4-phenylbutyrate on the vision of gja8 crispants.}

We injected embryos with buffer or Cas9 enzyme and a sgRNA targeted against gja8.L and gja8.S (sgRNA \#1). We allowed them to develop until stage 44, and we incubated half of them for 48h in 4PBA. We led them recover overnight in water before testing their vision as in Figure 2G. 

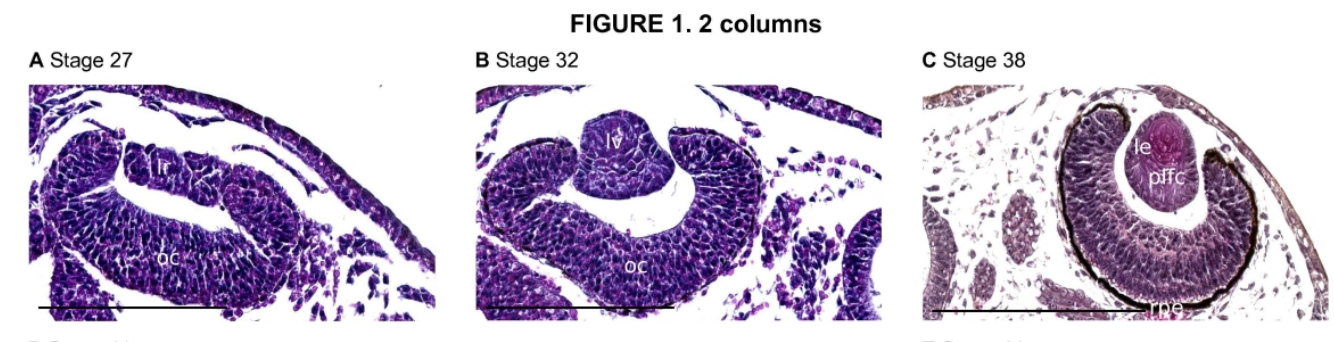

$$
\text { D Stage } 41
$$

$D^{\prime}$
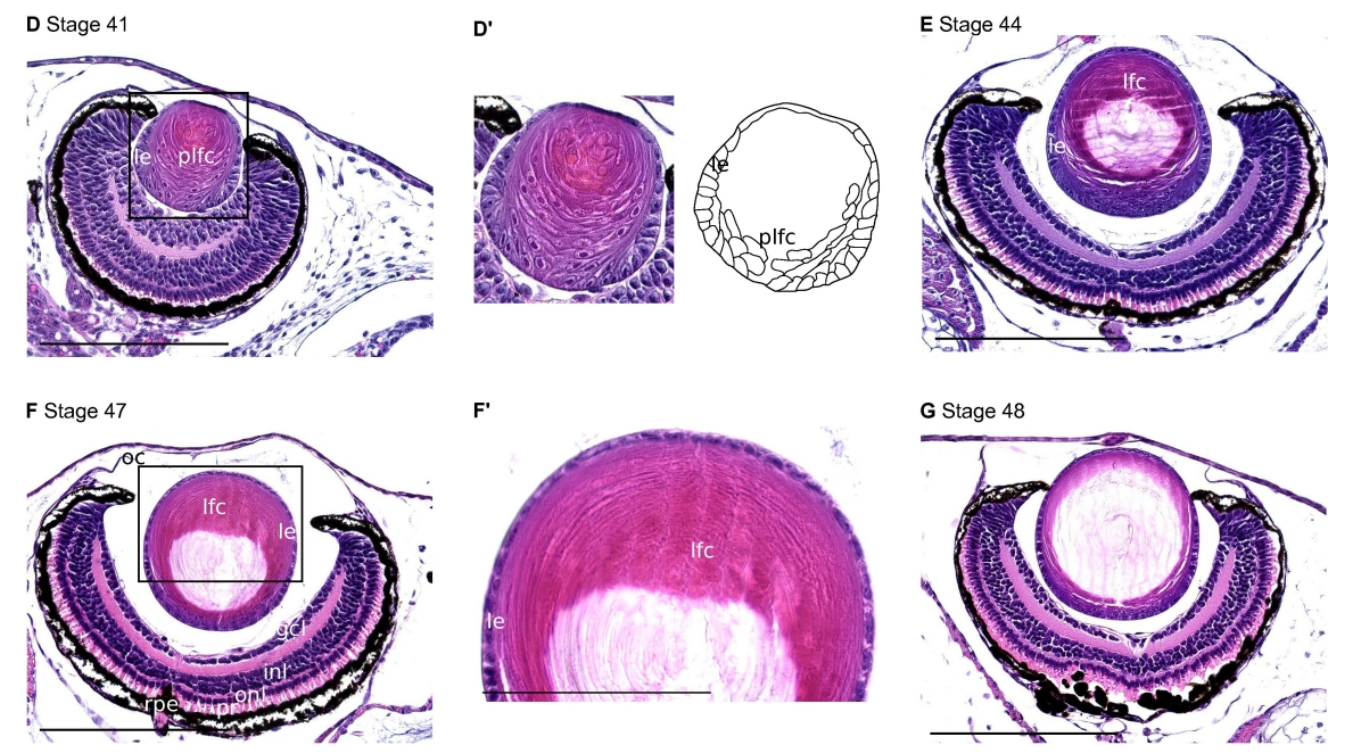

morphologically normal zebrafish tetraploids.

4. Diploidization of haploids by transient heat shocks during the teratogenic heat resilient windows aids in effective generation of gynogenic diploids.

\section{Funded By:}

1) Wellcome Trust Department of Biotechnology India Alliance (Intermediate Fellowship to Sreelaja Nair). Grant Number 13X301

2) Tata Institute of Fundamental Research (Department of Atomic Energy Government of India). Grant Number 12P0127

Accepted Articles are accepted, unedited articles for future issues, temporarily published online in advance of the final edited version.

(c) 2018 Wiley Periodicals, Inc.

Received: Oct 14, 2017; Revised: Apr 23, 2018; Accepted: May 12, 2018 


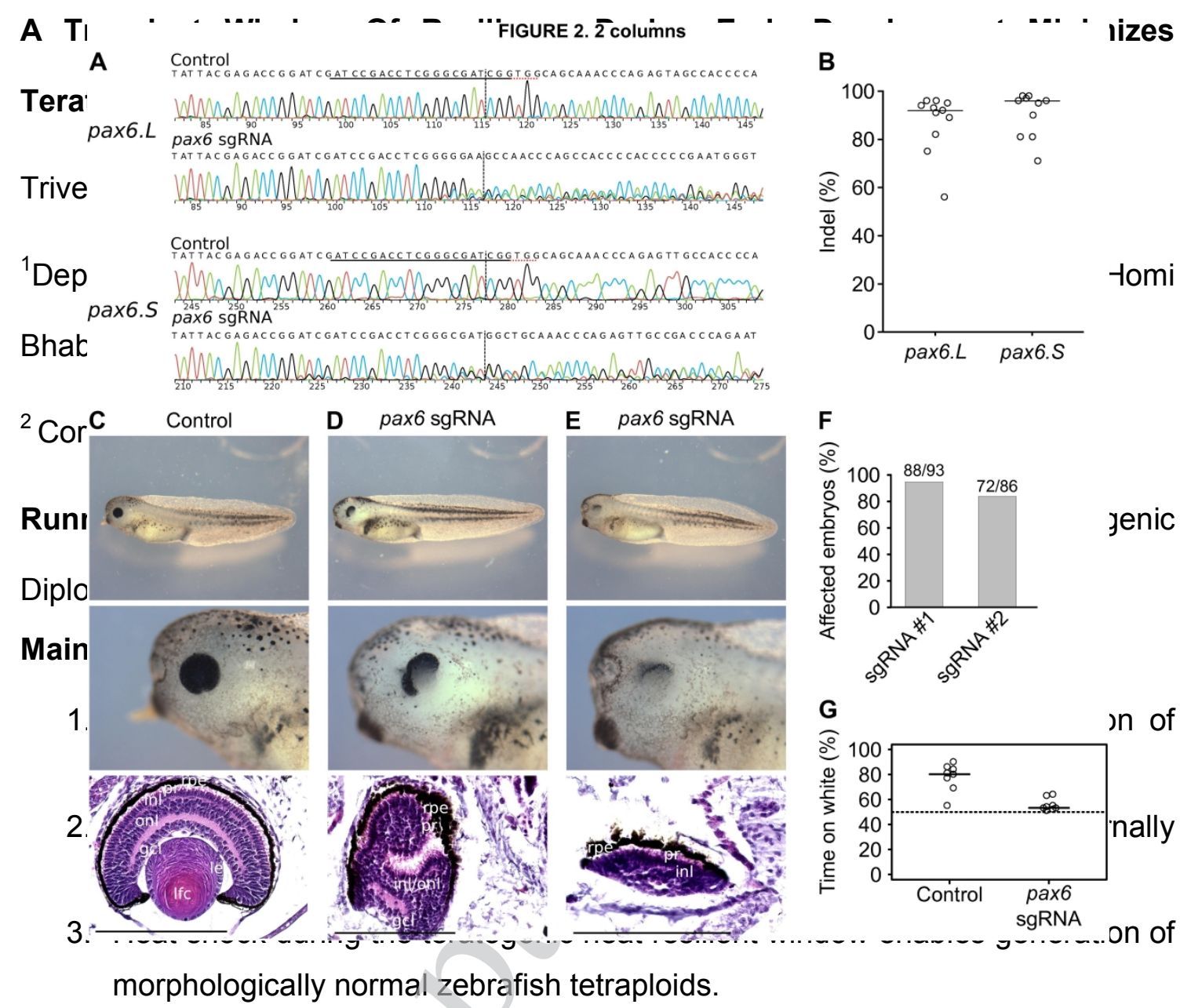

4. Diploidization of haploids by transient heat shocks during the teratogenic heat resilient windows aids in effective generation of gynogenic diploids.

\section{Funded By:}

1) Wellcome Trust Department of Biotechnology India Alliance (Intermediate Fellowship to Sreelaja Nair). Grant Number 13X301

2) Tata Institute of Fundamental Research (Department of Atomic Energy Government of India). Grant Number 12P0127

Accepted Articles are accepted, unedited articles for future issues, temporarily published online in advance of the final edited version.

(c) 2018 Wiley Periodicals, Inc.

Received: Oct 14, 2017; Revised: Apr 23, 2018; Accepted: May 12, 2018 
FIGURE 3 ( 2 columns)

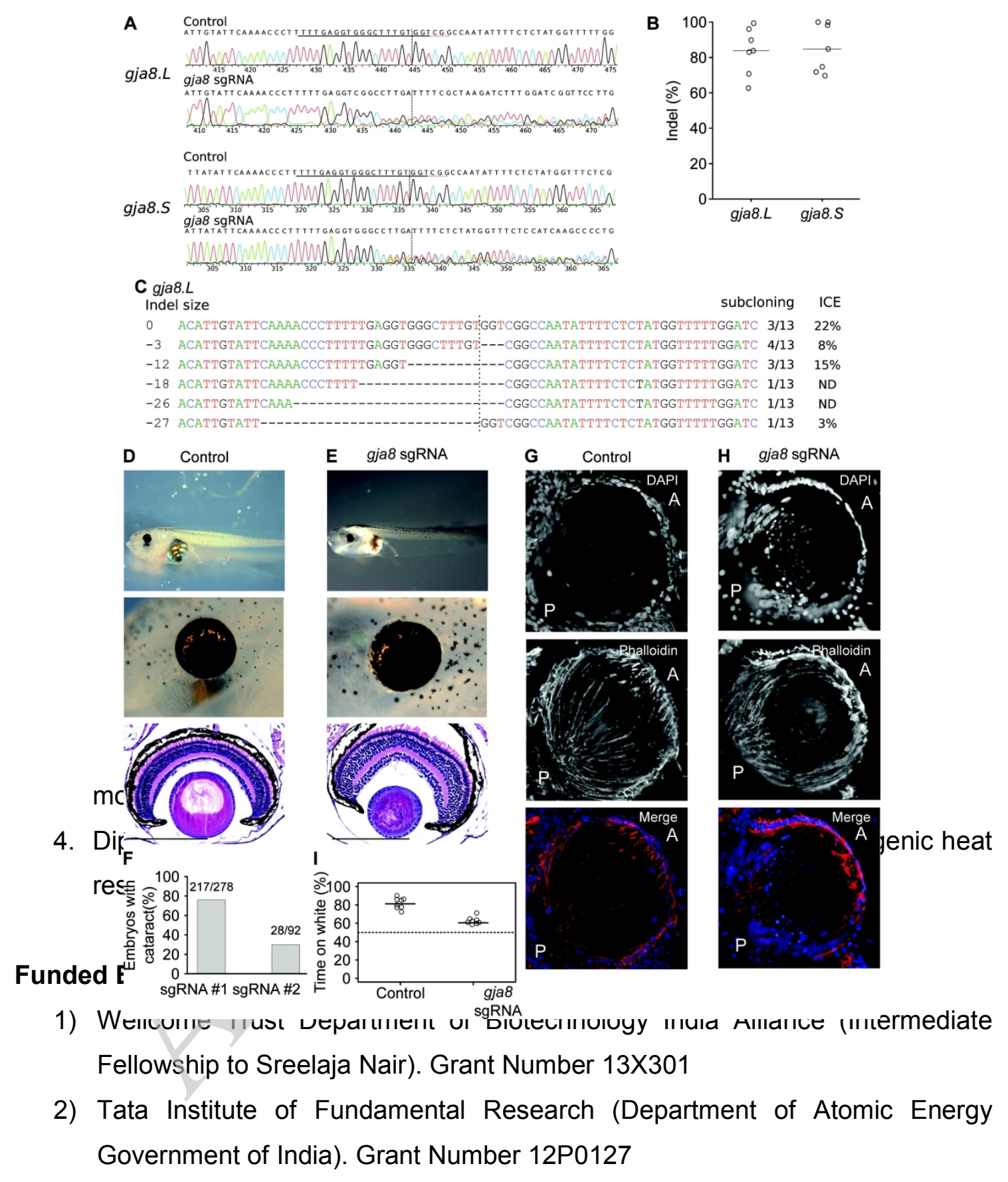

Government of India). Grant Number 12P0127

Accepted Articles are accepted, unedited articles for future issues, temporarily published online in advance of the final edited version.

(C) 2018 Wiley Periodicals, Inc.

Received: Oct 14, 2017; Revised: Apr 23, 2018; Accepted: May 12, 2018 
FIGURE 4. 1.5 column
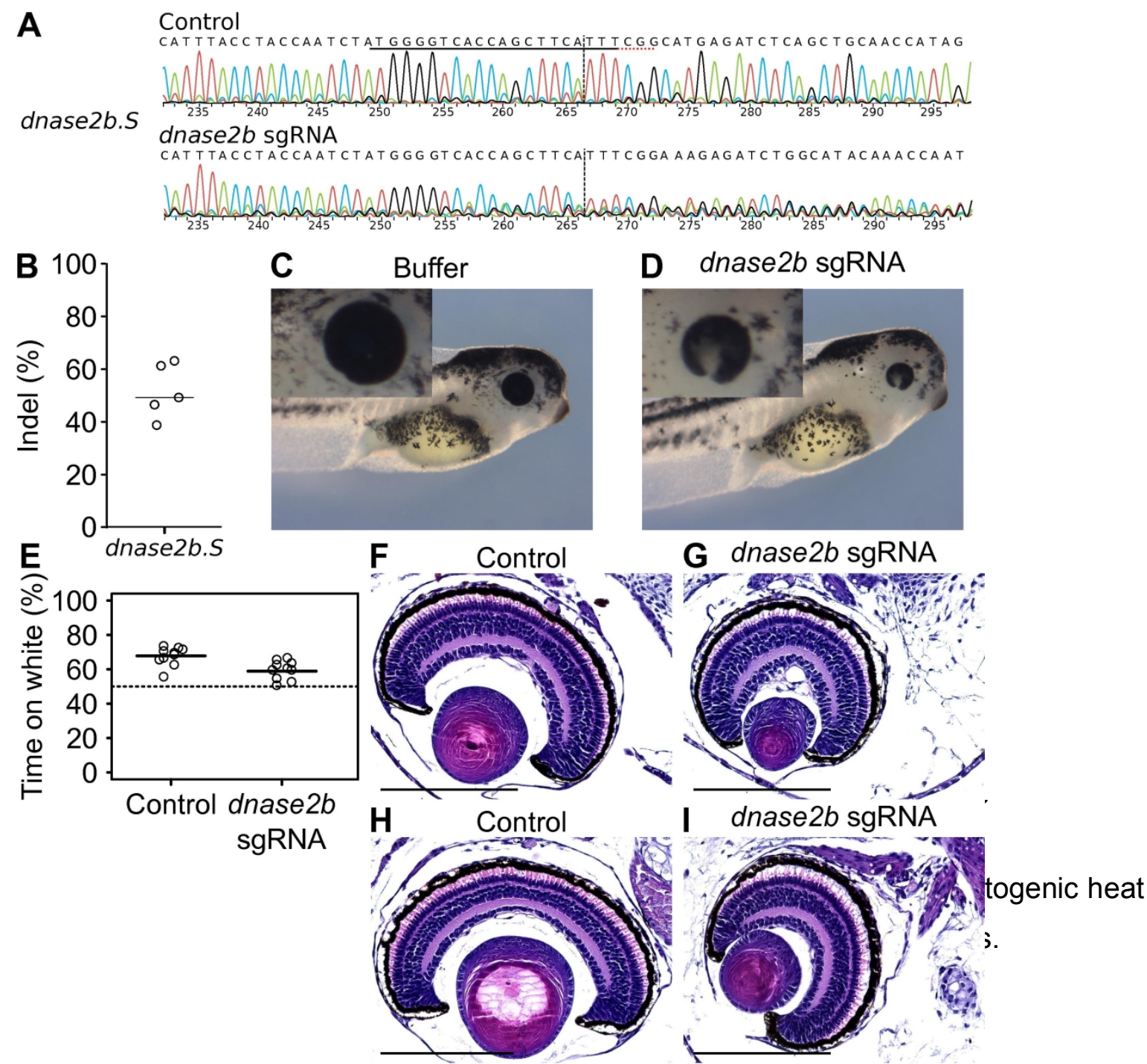

1) Wellcome Trust Department of Biotechnology India Alliance (Intermediate Fellowship to Sreelaja Nair). Grant Number 13X301

2) Tata Institute of Fundamental Research (Department of Atomic Energy Government of India). Grant Number 12P0127

Accepted Articles are accepted, unedited articles for future issues, temporarily published online in advance of the final edited version.

(C) 2018 Wiley Periodicals, Inc.

Received: Oct 14, 2017; Revised: Apr 23, 2018; Accepted: May 12, 2018 


\section{Figure 5, 1 column}

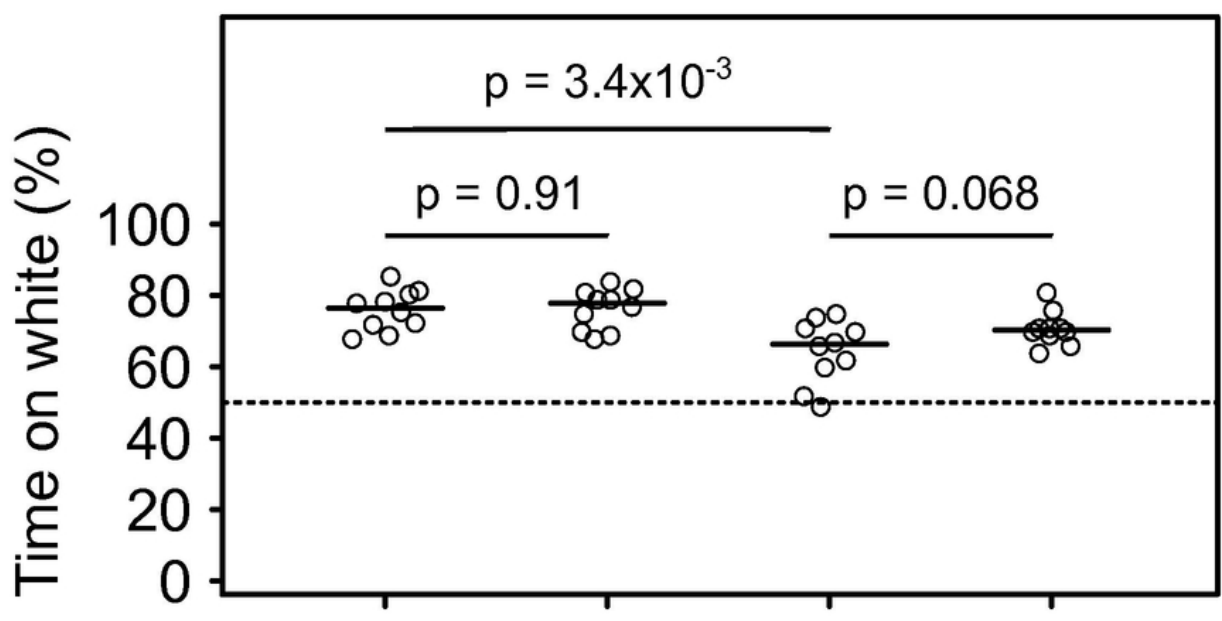

$\begin{array}{ccccc}\text { Injection } & \text { Buffer } & \text { Buffer } & \begin{array}{c}\text { gja8 } \\ \text { sgRNA }\end{array} & \begin{array}{c}\text { gja8 } \\ \text { sgRNA }\end{array} \\ \text { Treatment } & \text { None } & \text { 4PBA } & \text { None } & \text { 4PBA }\end{array}$

3. Heat shock during the teratogenic heat resilient window enables generation of morphologically normal zebrafish tetraploids.

4. Diploidization of haploids by transient heat shocks during the teratogenic heat resilient windows aids in effective generation of gynogenic diploids.

\section{Funded By:}

1) Wellcome Trust Department of Biotechnology India Alliance (Intermediate Fellowship to Sreelaja Nair). Grant Number 13X301

2) Tata Institute of Fundamental Research (Department of Atomic Energy Government of India). Grant Number 12P0127

Accepted Articles are accepted, unedited articles for future issues, temporarily published online in advance of the final edited version.

(c) 2018 Wiley Periodicals, Inc.

Received: Oct 14, 2017; Revised: Apr 23, 2018; Accepted: May 12, 2018 